\title{
Brain-derived neurotrophic factor expression in dorsal root ganglia of a lumbar spinal stenosis model in rats
}

\author{
QINLIANG LI ${ }^{1}$, YI LIU ${ }^{1}$, ZHAOMING CHU ${ }^{1}$, JINCHUAN CHEN $^{1}$, FENGLEI DAI ${ }^{2}$, \\ XIAORONG ZHU ${ }^{3}$, ANKANG $\mathrm{HU}^{3}$ and CAI YUN ${ }^{4}$ \\ ${ }^{1}$ Department of Spine, The Affiliated Lianyungang Hospital of Xuzhou Medical College, Lianyungang, Jiangsu 222000; \\ ${ }^{2}$ Department of Spine, Nanjing Liuhe District People's Hospital, Nanjing, Jiangsu 210000; ${ }^{3}$ Experimental Animal Center, \\ Xuzhou Medical College, Xuzhou, Jiangsu 221000; ${ }^{4}$ Department of Orthopaedic, \\ Shijingshan Hospital of Beijing City, Beijing 100043, P.R. China
}

Received April 3, 2013; Accepted September 26, 2013

DOI: $10.3892 / \mathrm{mmr} .2013 .1723$

\begin{abstract}
This study aimed to investigate the expression of brain-derived neurotrophic factor (BDNF) in dorsal root ganglia (DRG) of a rat model of lumbar spinal stenosis (LSS). Adult male rats were divided into the operation and sham operation groups. The operation group was comprised of the rat models of LSS. Walking distance and BDNF expression levels in DRG were measured in the two groups at different time points. The total BDNF protein levels and positive cell mean optical density (MOD) values in the operation group were significantly higher at each time point compared with that of the sham operation and preoperative control groups $(\mathrm{P}<0.05)$. The total BDNF protein levels and MOD values following sport in the operation group were significantly higher compared with those prior to sport $(\mathrm{P}<0.05)$. In the sham operation group, BDNF protein levels and MOD values before and after sport at each time point showed no significant differences than those of the operation group $(\mathrm{P}>0.05)$. Moreover, BDNF protein levels and MOD values in the operation group indicated a negative correlation with walking distance. The present study demonstrated that the expression of BDNF in rat models of LSS increased with time and was associated with a decrease in walking distance. BDNF was therefore important for the process of intermittent claudication caused by LSS.
\end{abstract}

\section{Introduction}

Lumbar spinal stenosis (LSS) is a medical condition in which the spinal canal narrows, compressing the spinal cord and

Correspondence to: Professor Cai Yun, Department of Orthopaedic, Shijingshan Hospital of Beijing City, No.24 Shijingshan Road, Beijing 100043, P.R.China

E-mail: liqinl@163.com

Key words: brain-derived neurotrophicfactor, lumbar spinal stenosis, neuropathic pain, dorsal root ganglia, rats nerves at the level of the lumbar vertebra. This is due to the common occurrence of lumbar spondylolisthesis, slipped disk, ligamentous thickening and spinal degeneration that develops with aging. With the development of imaging methods and the increased aging of society, the incidence of LSS has shown an annual increase. LSS is one of the main causes of leg pain in older individuals (1-4), including intermittent claudication, numbness of buttocks and legs, activity disorder of waist and legs, muscle wasting and tendon dysreflexia (5). Intermittent claudication caused by neuropathic pain (NP) is a special chief complaint of LSS, which may be reconstructed by clinical workers; however, its mechanisms remain unclear. It is now commonly accepted that NP is associated with coccygeal nerve regional pathology, regional blood flow, neuroelectrical physiological changes, and varied compression on the nerves or spinal cord (6-10). Dorsal root ganglia (DRG) are the primary neurons of afferent pain perception that synthesize diverse control factors, and are a key relay station linking the peripheral and central nervous systems. DRG are involved in transmitting algesia and have important significance for the study of NP (11-15).

It has been established that a number of bioactive compounds participate in NP, such as brain-derived neurotrophic factor (BDNF), glial cell line-derived neurotrophic factor (GDNF), P substance, cyclooxygenase 2 (COX2), prostaglandin E2 (PGE2) and IE-6 (16,17). Moreover, previous studies $(18,19)$ demonstrated that neurotrophic factors are important for neuronal growth, maturation and the restoration of damage, including neuronal survival, axon growth, synaptic plasticity, transmission of the neuronal signal and restoration of nerve injury. Simultaneously, neurotrophic factors have been studied as neurotransmitters that are involved in the process of pain creation, control and restoration of nerve injury (20-25). Thereby, we suggested that an increased BDNF expression in DRG of rat models of LSS attenuates NP and results in intermittent claudication.

The aim of the present study was to investigate the expression of BDNF in DRG of rat models of LSS, and to evaluate the correlation between BDNF expression and flat plate walking distance. In addition, this study aimed to demonstrate that BDNF is important for the processes of intermittent claudication caused by LSS and NP. 


\section{Materials and methods}

Animals and grouping. Healthy adult male Sprague-Dawley rats ( $\mathrm{n}=136$; weight, 230-280 g) were purchased from the Animal Test Center of Xuzhou Medical University [Lianyungang, China; animal production license no. SCXK (su) 2012011-0003 and animal usage license no. SYXK (su) 2010-0011]. This study was performed in strict accordance with the recommendations in the Guide for the Care and Use of Laboratory Animals of the National Institutes of Health. The animal use protocol was reviewed and approved by the Institutional Animal Care and Use Committee of the Affiliated Lianyungang Hospital of Xuzhou Medical College (Lianyungang, China). All the animals had access to food and water ad libitum, lived in natural light with a temperature of $24 \pm 1^{\circ} \mathrm{C}$ and all testing personnel were qualified to experiment on animals. Eight rats were selected at random prior to surgery and used as the conjunctive control, the remaining rats were randomly divided into the LSS $(n=64)$ and sham operation $(n=64)$ groups. The animals undertook flat plate sports every day for 3 days prior to surgery. All the rats in the control group were sacrificed prior to surgery and enzyme-linked immunosorbent assay (ELISA) and immunohistochemistry were performed on four of these rats to measure BDNF content and expression. If animals died during the test process then additional animals were added to maintain the original test number.

LSS model building. Silica gel slices were made from 18FR two cavity pure silicone catheter for single use (C. R. Bard, Inc., Covington, GA, USA). A suitable section of silica gel slices were selected and cut into thin slices (length, $4 \mathrm{~mm}$; depth, $1.25 \mathrm{~mm}$ and thickness, $1 \mathrm{~mm}$ ). The outer tip showed a wedge shape and the border was rubbed blunt using abrasive paper (Huifeng abrasive paper Ltd., Guangzhou, China). The silica gel slices were then soaked in $75 \%$ alcohol for more than $30 \mathrm{~min}$.

For the operation group, rats were anesthetized with $10 \%$ chloral hydrate via intraperitoneal injection $(0.5 \mathrm{ml} / 100 \mathrm{~g}$, additional anesthetic was provided as required). At the two sides of the iliac crest, horizontally level to the L5 vertebra, we performed shaving and skin preparation and $75 \%$ alcohol was used for skin degerming. A median incision was made behind the Processus spinosus, the skin and subcutaneous fascia were cut in turn, and the paravertebral muscles were located according to joint motion between L6-S1 and separated. In addition, the supra- and interspinal ligaments of L4-L6 were cut, the S1 Processus spinosus was lifted using forceps and epidural tissues under the lamina of vertebra were separated to expose Dura mater spinalis. Furthermore, the silica gel slice was longitudinally embedded under the L5 lamina of vertebra. At this time, we observed shortened feet in the lower extremities and a tail-flick, which confirmed the accurate location and compression of the spinal cord. Therefore, we removed L5 Processus spinosus, lifted L4 Processus spinosus and the silica gel slice was longitudinally embedded using the previous method. The wound was rinsed with physiological saline and 40 units of gentamicin sulfate were intravenously administerd. The fascia and skin were sutured in turn.

For the sham operation group, we performed the same process as that of the operation group; but, the silica gel slice was not embedded.
Walking distance measurement. The walking distance was measured using a flat plate sport instrument (SportsArt Fitness Industry Corporation,Taiwan, China). All rats undertook flat plate sports 3 days prior to and following surgery until they were sacrificed. The walking speed began at $0.3 \mathrm{~m} /$ sec and accelerated to $0.5,0.8$ and $0.9 \mathrm{~m} / \mathrm{sec}$ every $5 \mathrm{~min}$. Measurements were recorded twice daily at the fixation time and the mean value was calculated. The walking distance was not recorded when rats had continuously fallen off the flat plate three times.

Computerized tomography $(C T)$ scan of rats. Four rats in the operation group were randomly selected for the CT scan (32 row spirals, GE Company, Fairfield, CT, USA; scan condition was $120.00 \mathrm{KV}, 200 \mathrm{MA}, 512 \times 512$ pixel; slice thickness, $0.625 \mathrm{~mm}$ and Dicom format). The scan result was imported into Materialise Mimics 13.0 finite element modeling software (Materialise Company, Leuven, Belgium) to construct finite element modeling of the lumbar in rats. Image-Pro Plus 6.0 (Media Cybernetics Company, PA, USA) was used to measure and calculate the sagittal and transection diameters of the spinal canal in L4 and L5 phase.

Sample clearage. PMSF crystal was added to PMSF solvents, and following dissolution and antigrading, $10 \mathrm{ml}$ $100 \mathrm{mM}$ PMSF was prepared. PMSF $(1 \mathrm{ml} 100 \mathrm{mM})$ was added to $100 \mathrm{ml} \mathrm{NP}-40$ and the NP-40 lysate of $1 \mathrm{mM}$ PMSF was obtained. The sample was defrosted at room temperature, and then cut into fragments and placed in a centrifuge tube. Lysate (200 $\mu \mathrm{l}$ ) was added to each centrifuge tube and following $1 \mathrm{~h}$ on ice the tissue homogenate was centrifuged $(10,000 \mathrm{x} \mathrm{g}$ at $4^{\circ} \mathrm{C}$ for $15 \mathrm{~min}$ ) and the supernatant was removed.

Determination of total protein content. The reagents were purchased from Promega Corporation (Fitchburg, WI, USA). Protein standard dispensing liquid $(0.8 \mathrm{ml})$ was added to $20 \mathrm{mg}$ protein standard solution (bovine serum albumin) following sufficient dissolution to give $25 \mathrm{mg} / \mathrm{ml}$ protein standard solution. Moreover, $20 \mu \mathrm{l}$ of $25 \mathrm{mg} / \mathrm{ml}$ protein standard solution was added to $980 \mu \mathrm{l}$ phosphate buffered-saline (PBS) and diluted to $0.5 \mathrm{mg} / \mathrm{ml}$ of the final content. BCA fluid was formed by mixing $0.8 \mathrm{ml} \mathrm{BCA}$ reagent $B$ and $40 \mathrm{ml}$ reagent $A$ at a ratio of 1:50. The protein standard solution was added into the 96-well plates at $0,1,2,4,8,12,16$ and $20 \mu 1,10 \mu \mathrm{l}$ supernatant and PBS were added to increase the concentration to $20 \mu \mathrm{l}$ in each well. In addition, $200 \mu \mathrm{l}$ BCA fluid was added into each well and the absorbance was measured at $37^{\circ} \mathrm{C}$ for $30 \mathrm{~min}$. The optical density (OD) value at $562 \mathrm{~nm}$ absorbance was assayed with ELISA, relative to OD 450, it was equal to OD 450 (sample) - OD 450 (control). Protein concentration of the samples was calculated using a standard curve, the practical BDNF concentration was the measurement result multiplied by two.

ELISA test. All reagents were purchased from Promega Corporation. The rats were sacrificed by administrating an overdosed level of anaesthetic, the L4-L5 bilateral nerve root was quickly separated and DRG were identified (four DRG were removed from each animal and considered as the same samples). Nerve fibers $(1 \mathrm{~cm})$, including DRG were cut and 
removed. The blood was washed with PBS and stored in the freezer at $-80^{\circ} \mathrm{C}$ until the ELISA test (Fig. 1).

BDNF protein standard liquid was prepared in the first $24 \mathrm{~h}$ after the animals were sacrificed. Sample dilution $(1 \mathrm{ml})$ was added to $10 \mathrm{ng}$ BDNF standard preparation tube and $10 \mathrm{ng} /$ $\mathrm{ml}$ BDNF protein standard fluid was obtained. Moreover, $0.2 \mathrm{ml} 10 \mathrm{ng} / \mathrm{ml}$ was added to $0.8 \mathrm{ml}$ sample dilution and $2000 \mathrm{pg} / \mathrm{ml}$ BDNF protein standard fluid was obtained. Six centrifuge tubes were marked and $0.3 \mathrm{ml}$ sample dilution was added to each centrifuge tube; $0.3 \mathrm{ml} 2000 \mathrm{pg} / \mathrm{ml}$ fluid was added into the first tube; and $0.3 \mathrm{ml}$ from the first tube was added into the second tube, with this procedure continuing in this manner. From the six tubes, $0.1 \mathrm{ml}$ protein standard fluid was added to a 96 -well plate, $0.1 \mathrm{ml}$ sample dilution was added to the first well as the blank, and $50 \mu 1$ sample supernatant and $50 \mu 1$ sample dilution were added to each empty well. The lid was added and the well plate was incubated for $90 \mathrm{~min}$ at $37^{\circ} \mathrm{C}$. Furthermore, $100 \mu \mathrm{l}$ BDNF antibody was added to $9.9 \mathrm{ml}$ antibody dilution (1:100) and $10 \mathrm{ml}$ BDNF antibody fluid was obtained. Following incubation, liquid in the well plate was removed and the plate was dried with absorbent paper as the drying process was slow. Antibody fluid $(0.1 \mathrm{ml})$ was added to each well and incubated for $60 \mathrm{~min}$ at $37^{\circ} \mathrm{C}$. The plate was washed three times with PBS (1 min per well) and liquid was removed and the plate was dried with absorbent paper. $\mathrm{ABC}$ and TMB fluids (Promega Corporation) were also incubated for $30 \mathrm{~min}$ at $37^{\circ} \mathrm{C}$. Furthermore, $100 \mu \mathrm{l} \mathrm{ABC}$ was added to $9.9 \mathrm{ml} \mathrm{ABC}$ dilution and $10 \mathrm{ml} \mathrm{ABC}$ fluid was obtained. Following the dilution, $0.1 \mathrm{ml} \mathrm{ABC}$ fluid was added into each well and incubated for $30 \mathrm{~min}$ at $37^{\circ} \mathrm{C}$. The plate was washed five times with PBS (Promega Corporation; 2 min per well), liquid was removed and the well plate was dried with absorbent paper. TMB coloration liquid $(90 \mu \mathrm{l})$ was added to the plate and incubated for $30 \mathrm{~min}$ at $37^{\circ} \mathrm{C}$. Additionally, $100 \mu \mathrm{l} \mathrm{TMB}$ stopping buffer was added into each well and the OD value of A450 was assessed using ELISA for $30 \mathrm{~min}$. Relative to OD 45O, it was equal to OD 450 (sample) - OD 450 (control). Protein content of the sample was calculated using a standard curve, practical BDNF concentration was the measured result multiplied by two.

BDNF content equaled the BDNF concentration divided by the BCA concentration (pg/mg total protein).

Immunohistochemistry. After the animals were sacrificed, $4 \%$ paraformaldehyde was quickly administered into the rat heart. DRG were removed using the previous method, soaked in $4 \%$ paraformaldehyde liquid and fixed. Following dehydration, clearing, routine paraffin embedding and $3-\mu \mathrm{m}$ serial sections, we performed immunohistochemical staining with streptavidin-peroxidase. DRG slices were heated at $60^{\circ} \mathrm{C}$ overnight, dewaxed in xylol and hydrated with gradient alcohol. To remove endogenous peroxidase, slices were incubated at $37^{\circ} \mathrm{C}$ for $10 \mathrm{~min}$ using $3 \% \mathrm{H}_{2} \mathrm{O}_{2}$. Then slices were washed three times for $5 \mathrm{~min}$ in PBS (pH 7.4). Slices were placed into sodium citrate liquid $(\mathrm{pH}$ 6.0) and heated to boiling by microwave for $2 \mathrm{~min}$. Slices were allowed to cool for $15 \mathrm{~min}$, then washed three times for $5 \mathrm{~min}$ with PBS. In addition, $10 \%$ normal goat serum confining liquid was added for $20 \mathrm{~min}$ at room temperature and any additional liquid was removed. Rabbit anti-rat BDNF polyclonal antibody
(50 $\mu \mathrm{l}$ ) (1:200; Abnova Corporation, CA, USA) was added overnight at $4^{\circ} \mathrm{C}$ and PBS replaced the antibody in the blank. Furthermore, slices were washed three times for $5 \mathrm{~min}$ with PBS, biotin-labeled goat anti-rabbit IgG (1:50) was added and slices were incubated for $30 \mathrm{~min}$ at $37^{\circ} \mathrm{C}$. Slices were then washed three times for $5 \mathrm{~min}$ with $\mathrm{PBS}$. Horseradish peroxidase labeling strepto-albumin stock solution (1:300) was added, incubated for $30 \mathrm{~min}$ at $37^{\circ} \mathrm{C}$ and slices were washed three time for 5 min with PBS. In addition, DRG slices were then stained with 3,3'-diaminobenzidine for $30 \mathrm{~min}$ and washed three times for $5 \mathrm{~min}$ with PBS before being stained again with hematoxylin and eosin. Slices were then dehydrated with alcohol gradient and cleared with xylol. DRG slices were mounted with neutral gum (Promega Corporation) and observed under a light microscope (magnification, x200). Photographs recorded the staining of the DRG slices. We used Image-Pro Plus software, version 6.0 to carry out the gradation analysis for DRG slices and calculate the mean optical density (MOD) of BDNF-positive action in DRG.

Statistical analysis. Data are expressed as the mean \pm standard deviation and analyzed with SPSS software, version 16.0 (SPSS Inc., Chicago, IL, USA). An independent sample t-test was used to compare BDNF content difference and flat plate sports distance in each group, between groups and before and after sports. Two- and one-way analysis of variance and multiple comparison methods were used to analyze BDNF expression changes between each group at all time-points and before and after sports. Bivariate correlation was used to analyze BDNF expression and sports distance. $\mathrm{P}<0.05$ was considered to indicate a statistically significant difference.

\section{Results}

Performance. The majority of rats in the operation and sham operation groups were able to walk 1 day post-surgery. Eight rats in the operation group and 1 rat in the sham operation group died during the experiment; therefore, animals were added to maintain the sample number. In the operation group, 7 rats were paralyzed in the lower extremities and showed crossing. Unilateral hind legs of five rats were paralysis and one rat was suspected to have an infection. All the rats in the operation group demonstrated drooping tails and were suspected to have uroclepsia or constipation. In the sham operation group, no obvious differences were observed in their walking abilities compared with those prior to surgery and no animals indicated infection.

Internal diameter measurement of the spinal canal. In the operation group, the sagittal diameter of the spinal canal in L4 phase was $1.31 \pm 0.05 \mathrm{~mm}$ and the transection diameter was $2.05 \pm 0.02 \mathrm{~mm}$. In the L5 phase, the sagittal diameter was $1.16 \pm 0.03 \mathrm{~mm}$ and transection diameter was $1.99 \pm 0.02 \mathrm{~mm}$. Rats were dissected and the lamina of vertebra was cut. The obvious compression of the coccygeal nerve was exposed and compression was $\sim 50-70 \%$.

BDNF content changes. BDNF total protein levels in the operation group were $202.06 \pm 89.25 \mathrm{pg} / \mathrm{mg}$ prior to surgery, which was significantly higher than that of the 
Table I. BDNF expression levels $(n=4)$, MOD $(n=4)$ and walking distance $(n=8)$ in each group.

\begin{tabular}{|c|c|c|c|c|c|}
\hline Post surgery & Group & & $\begin{array}{c}\text { BDNF } \\
(\mathrm{pg} / \mathrm{mg} \text { total protein })\end{array}$ & $\begin{array}{r}\text { MOD } \\
( \pm s)\end{array}$ & $\begin{array}{l}\text { Sports distance } \\
\text { (m) }\end{array}$ \\
\hline & Control prior to surgery & & $114.28 \pm 10.78$ & $0.199 \pm 0.028$ & $395.76 \pm 32.79$ \\
\hline \multirow[t]{6}{*}{1 day } & Operation & Before sport & $147.22 \pm 32.31$ & $0.265 \pm 0.021$ & $42.67 \pm 38.01$ \\
\hline & & After sport & $218.27 \pm 56.90$ & $0.283 \pm 0.031$ & - \\
\hline & & Sum $(n=8)$ & $182.75 \pm 57.25^{\mathrm{a}}$ & $0.273 \pm 0.026^{\mathrm{a}}$ & - \\
\hline & Sham operation & Before sport & $123.20 \pm 9.84$ & $0.201 \pm 0.021$ & $75.00 \pm 39.19$ \\
\hline & & After sport & $120.16 \pm 65.84$ & $0.198 \pm 0.022$ & - \\
\hline & & Sum $(n=8)$ & $121.68 \pm 43.61$ & $0.199 \pm 0.021$ & - \\
\hline \multirow[t]{6}{*}{3 days } & Operation & Before sport & $234.03 \pm 116.08$ & $0.298 \pm 0.019$ & $62.63 \pm 31.69^{\mathrm{e}}$ \\
\hline & & After sport & $295.83 \pm 100.08$ & $0.332 \pm 0.016^{\mathrm{d}}$ & - \\
\hline & & Sum $(n=8)$ & $264.93 \pm 105.64^{\mathrm{a}, \mathrm{b}}$ & $0.316 \pm 0.025^{\mathrm{a}, \mathrm{c}}$ & - \\
\hline & Sham operation & Before sport & $125.17 \pm 9.80$ & $0.202 \pm 0.022$ & $183.66 \pm 12.13$ \\
\hline & & After sport & $127.5 \pm 24.36$ & $0.197 \pm 0.019$ & - \\
\hline & & Sum $(n=8)$ & $126.36 \pm 17.23$ & $0.199 \pm 0.019$ & - \\
\hline \multirow[t]{6}{*}{7 days } & Operation & Before sport & $168.12 \pm 28.75$ & $0.293 \pm 0.029$ & $100.13 \pm 68.47^{\mathrm{e}}$ \\
\hline & & After sport & $271.90 \pm 118.14$ & $0.314 \pm 0.031$ & - \\
\hline & & Sum $(n=8)$ & $220.01 \pm 97.02^{\mathrm{a}}$ & $0.303 \pm 0.030^{\mathrm{a}}$ & - \\
\hline & Sham operation & Before sport & $122.44 \pm 29.62$ & $0.201 \pm 0.028$ & $292.55 \pm 99.27$ \\
\hline & & After sport & $116.68 \pm 38.47$ & $0.201 \pm 0.019$ & - \\
\hline & & Sum $(n=8)$ & $120.56 \pm 31.84$ & $0.201 \pm 0.022$ & - \\
\hline \multirow[t]{6}{*}{14 days } & Operation & Before sport & $127.51 \pm 35.91$ & $0.273 \pm 0.047$ & $82.69 \pm 58.93^{\mathrm{e}}$ \\
\hline & & After sport & $153.57 \pm 49.28$ & $0.291 \pm 0.037$ & - \\
\hline & & Sum $(n=8)$ & $140.54 \pm 42.27$ & $0.282 \pm 0.041^{\mathrm{a}}$ & - \\
\hline & Sham operation & Before sport & $121.57 \pm 51.24$ & $0.197 \pm 0.034$ & $279.34 \pm 106.10$ \\
\hline & & After sport & $125.07 \pm 57.06$ & $0.204 \pm 0.014$ & - \\
\hline & & Sum $(n=8)$ & $123.41 \pm 50.23$ & $0.201 \pm 0.024$ & - \\
\hline
\end{tabular}

${ }^{\mathrm{a}} \mathrm{P}<0.05$ brain-derived neurotrophic factor (BDNF) expression levels in the operation group was significantly higher at all time points before and after surgery compared with those in the sham operation and control groups. ${ }^{b} \mathrm{P}<0.05 \mathrm{BDNF}$ expression levels were significantly higher compared with those in the sham operation group; ${ }^{\mathrm{C}} \mathrm{P}<0.05 \mathrm{MOD}$ was significantly higher in the operation group on the day 3 postoperatively compared with that of the sham operation group. There were significant differences at 1 and 14 days following surgery. ${ }^{\mathrm{d}} \mathrm{P}<0.05 \mathrm{At}$ the same time-points between before and after sports and ${ }^{\mathrm{e}} \mathrm{P}<0.05$ walking distance was significantly lower compared with that at the same time points in the sham operation group. MOD, mean optical density.

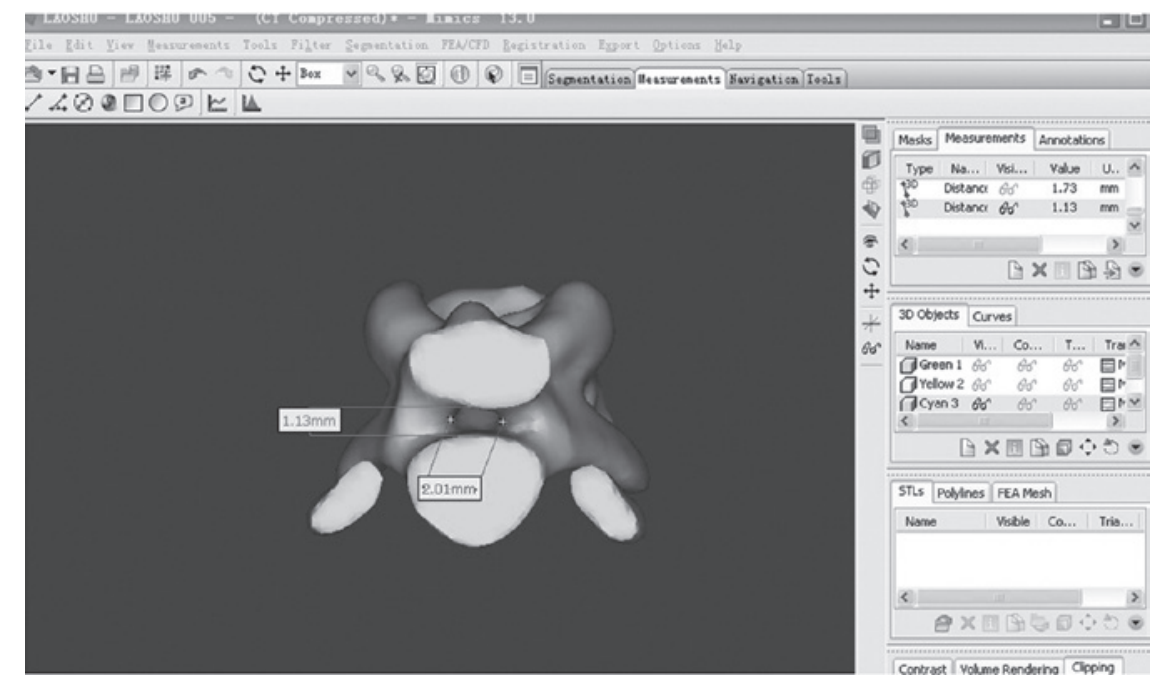

Figure 1. Measurement of the transection and sagittal diameters of the spinal canal. 
Table II. BDNF expression levels before and after sports $( \pm s)$ and MOD ( \pm s) in each group.

\begin{tabular}{|c|c|c|c|}
\hline Group & Prior to and after sports & $\begin{array}{c}\text { BDNF content } \\
\text { (pg/mg total protein) }\end{array}$ & MOD \\
\hline \multirow[t]{3}{*}{ Operation } & Before $(n=16)$ & $169.22 \pm 70.99$ & $0.281 \pm 0.031$ \\
\hline & After $(n=16)$ & $234.90 \pm 95.50^{\mathrm{a}}$ & $0.305 \pm 0.033^{\mathrm{a}}$ \\
\hline & Sum $(n=32)$ & $202.06 \pm 89.25^{\mathrm{b}}$ & $0.293 \pm 0.034^{\mathrm{b}}$ \\
\hline \multirow[t]{3}{*}{ Sham operation } & Before $(n=16)$ & $123.14 \pm 27.22$ & $0.200 \pm 0.024$ \\
\hline & After $(n=16)$ & $122.86 \pm 44.12$ & $0.199 \pm 0.017$ \\
\hline & Sum $(n=32)$ & $123.00 \pm 36.06$ & $0.200 \pm 0.206$ \\
\hline Control prior to surgery $(n=4)$ & - & $114.28 \pm 10.78$ & $0.199 \pm 0.028$ \\
\hline
\end{tabular}

${ }^{\mathrm{a}} \mathrm{P}<0.05$ compared with that before sports and ${ }^{\mathrm{b}} \mathrm{P}<0.05$ compared with that in the sham operation and control groups prior to surgery. BDNF, brain-derived neurotrophic factor; MOD, mean optical density.

A

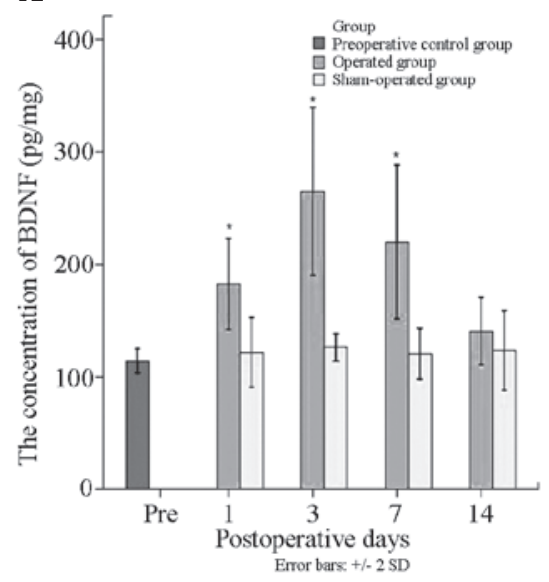

B

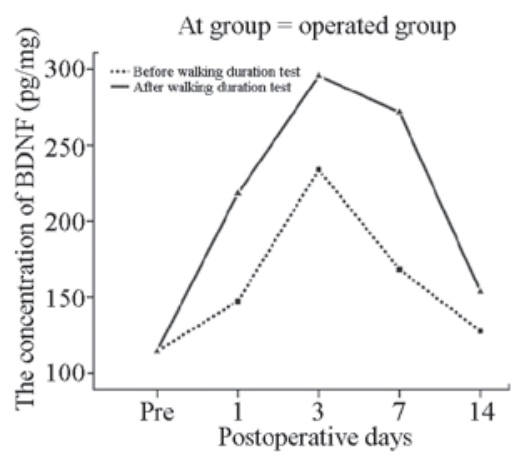

C

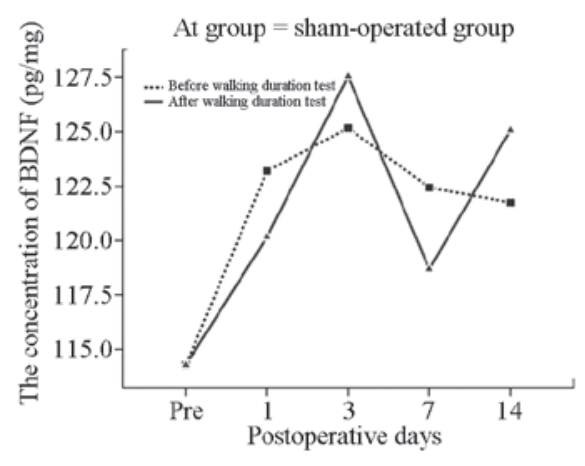

Figure 2. Measurement of BDNF expression levels in each group at all time points by ELISA. (A) * P $<0.05$ at 1,3 and 7 days following surgery in the operation group compared with that in the sham operation and control groups prior to surgery. (B) BDNF expression levels in the operation group increased rapidly postoperatively. An additional increase was observed following sport than before sport. BDNF expression peaked on the third day, then gradually decreased. (C) BDNF expression levels showed no significant differences between before and after sports at each time point in the sham operation group. BDNF, brain-derived neurotrophic factor; ELISA, enzyme-linked immunosorbent assay.

sham operation $(123.00 \pm 36.06 \mathrm{pg} / \mathrm{mg})$ and control groups $(114.28 \pm 10.78 \mathrm{pg} / \mathrm{mg})(\mathrm{P}<0.05)$. There were no differences between the sham operation and the control groups prior to surgery $(\mathrm{P}>0.05)$.

In the operation group, BDNF total protein concentration before and after sports was $169.22 \pm 70.99 \mathrm{pg} / \mathrm{mg}$ and $234.90 \pm 95.50 \mathrm{pg} / \mathrm{mg}$, respectively $(\mathrm{P}<0.05)$. There were significant differences at each time point in the operation group $(\mathrm{P}<0.05)$, BDNF total protein levels were highest 3 days following surgery and reached $264.93 \pm 105.64 \mathrm{pg} / \mathrm{mg}$. Moreover, there were significant differences prior to surgery compared with that 14 days following surgery $(140.54 \pm 42.27)$ $(\mathrm{P}<0.05)$. There were no significant differences at the remaining time points $(\mathrm{P}>0.05)$.

BDNF content following sports in the operation group at the same time points was higher than that prior to sports, but there were no significant differences $(P>0.05)$. Values before and after sports in the sham operation group at each time point showed no significant differences $(\mathrm{P}>0.05)$ (Tables I and II, Fig. 2).
$B D N F$ protein expression changes. In the operation group, BDNF protein expression was upregulated 1 day following surgery. A positive reaction was distributed in each type of neuron, but it was mainly distributed in the intracytoplasm of neurons in the middle of DRG with a small diameter (10-50 $\mu \mathrm{m})$. BDNF distribution was also observed in nerve fibers and marginal expression was identified in the nucleus (Fig. 3).

MOD in the operation group $(0.293 \pm 0.034)$ was significantly higher than that in the sham operation $(0.200 \pm 0.206)$ and control groups prior to surgery $(0.199 \pm 0.028)(\mathrm{P}<0.05)$. There were no differences between the sham operation and control groups $(\mathrm{P}>0.05)$. In the operation group, the MOD values before $(0.281 \pm 0.031)$ and after sports $(0.305 \pm 0.033)$ were significantly different $(\mathrm{P}<0.05)$. Multiple comparison with the SNK method demonstrated significant differences at each time point in the operation group $(\mathrm{P}<0.05)$. MOD was the highest 3 days post surgery $(0.316 \pm 0.025)$ and lowest 1 day post surgery $(0.273 \pm 0.026)(\mathrm{P}<0.05)$. There were no differences at the remaining time points. 

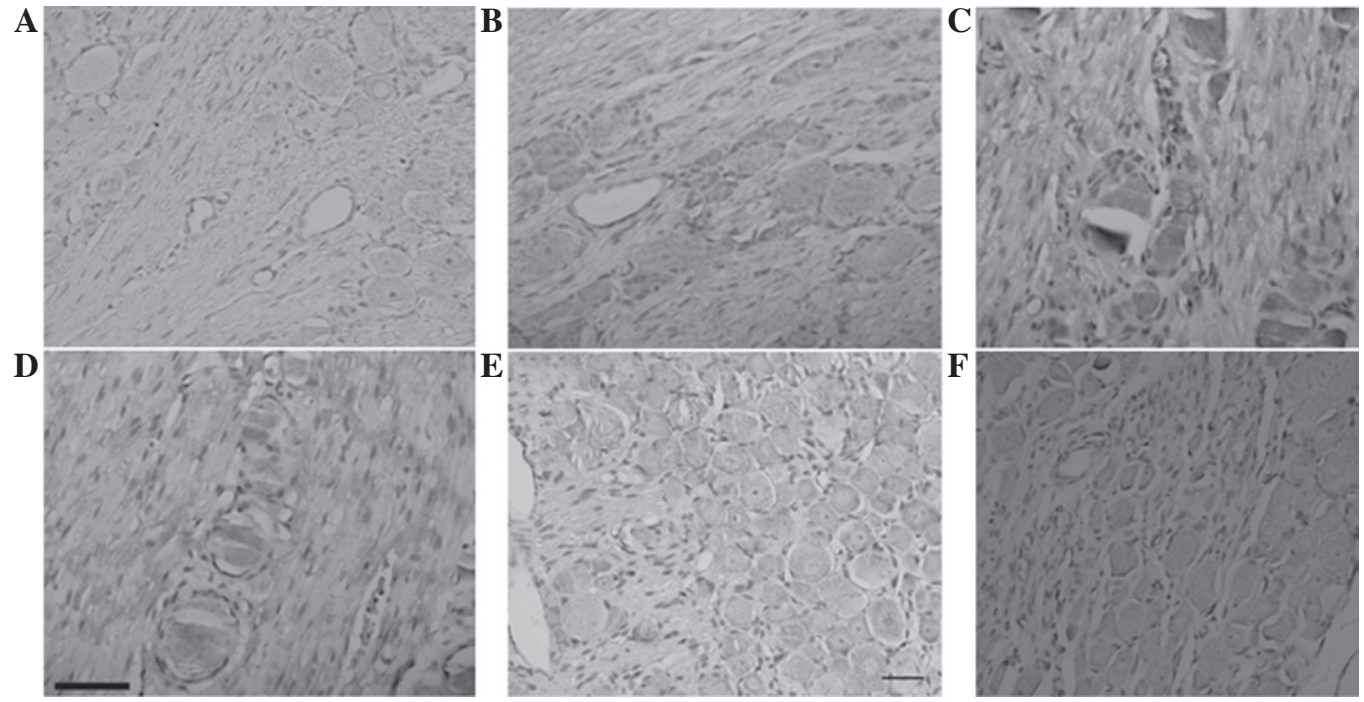

Figure 3. BDNF expression change tested by immunohistochemisty (streptavidin-peroxidase staining; magnification, x200). BDNF expression in the operation group at (A) 1, (B) 3, (C) 7 and (D) 14 days following surgery. BDNF expression levels peaked 3 days following surgery; (B) BDNF expression showed significant differences compared with that in the (E) sham operation and (F) control groups prior to surgery. Scale bar: $50 \mu \mathrm{m}$. BDNF, brain-derived neurotrophic factor.

A

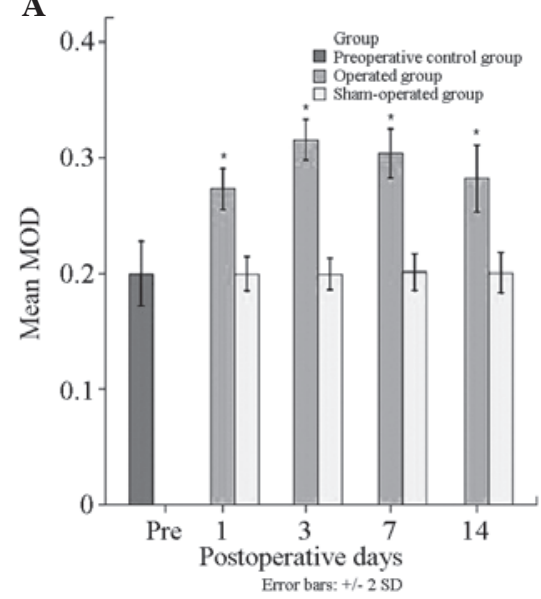

B

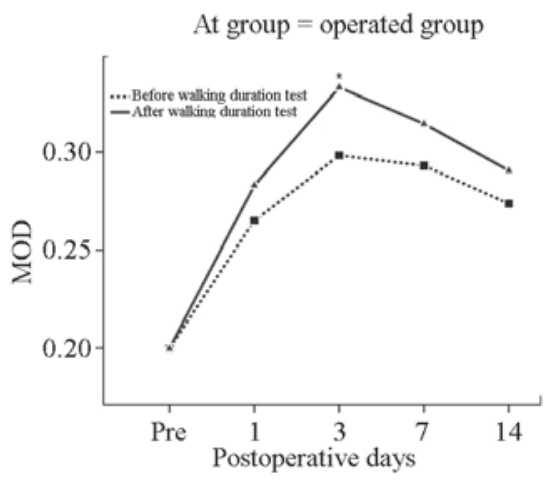

C

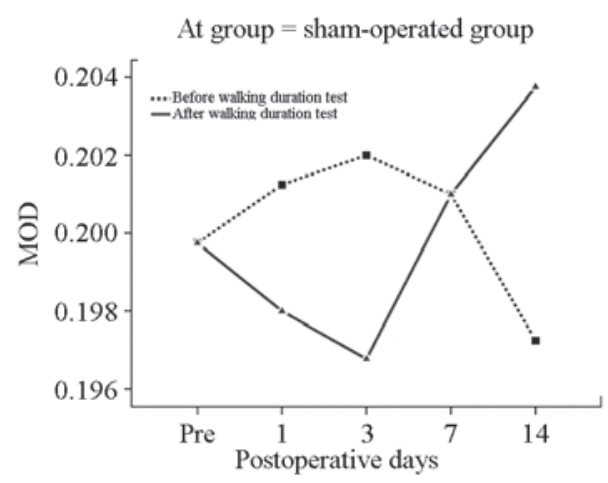

Figure 4. Immunohistochemistry examined BDNF expression levels at each time point in each group. (A) There were significant differences at $1,3,7$ and 14 days following surgery in the operation group compared with that in the sham operation and control groups prior to surgery ("P<0.05). (B) MOD in the operation group following surgery rapidly increased and then increased following sports compared with that before sports. BDNF expression levels peaked on day 3 and then decreased gradually, with a signifcant difference being indicated between before and after sports ( $\mathrm{P}<0.05)$. (C) MOD showed no significant differences between before and after sports at each time point in the sham operation group. BDNF, brain-derived neurotrophic factor; MOD, mean optical density.

In the operation group, the MOD following surgery was significantly higher after sports than before sports. However, t-test analysis compared the same time points before and after sports and demonstrated that 3 days following surgery BDNF expression levels after sports were significantly different than those before sports $(\mathrm{P}<0.05)$. In the sham operation group, MOD at the same time points before and after sports showed no significant differences $(\mathrm{P}>0.05)$ (Tables I and II; Fig. 4).

Correlation between the distance measured following flat plate sports and BDNF expression levels. In the operation group, flat plate sports distance following surgery was significantly less than that before surgery $(\mathrm{P}<0.05)$. In the sham operation group before and after surgery, walking distance was not significantly different. Walking distance following surgery in the operation group was significantly less than that in the sham operation group $(\mathrm{P}<0.05)$ (Table I, Fig. 5).

ELISA confirmed the results of the immunohistochemical staining in the operation group. BDNF expression levels showed a significant negative correlation with walking distance (correlation coefficient $\mathrm{r}=-0.05$ and $-0.65, \mathrm{P}<0.05$ ); however, the correlativity was not close (Fig. 6).

\section{Discussion}

There are a number of methods that can be used to prepare a rat model of LSS, such as use of a metal device, sacculus, composite material, lamina of vertebrae degeneration osteophyte and tumor implantation (26-31). Spinal stenosis caused by silica gel embedding is similar to spinal stenosis as a result 


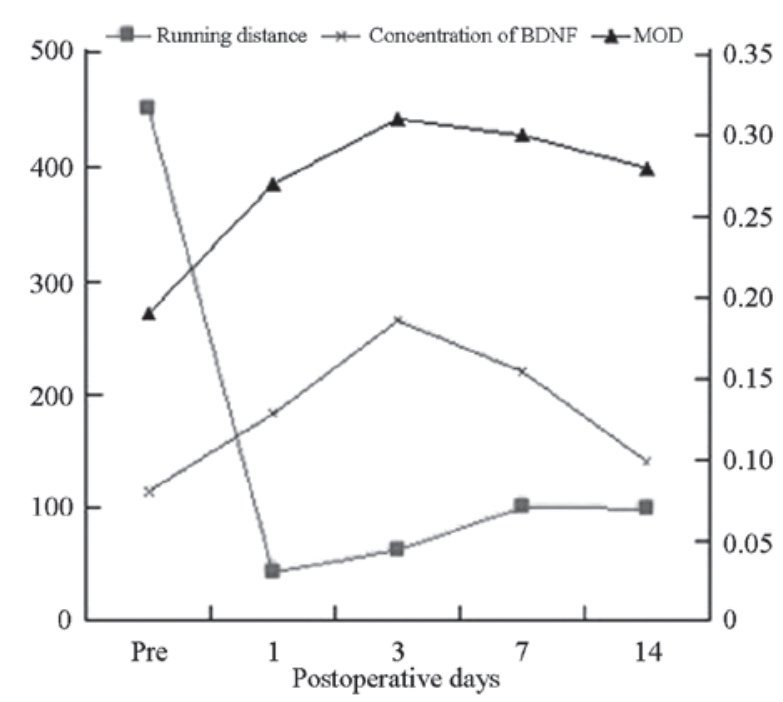

Figure 5. Walking distance between each group at all time points. Walking distance decreased rapidly 1 day following surgery in the two groups. By contrast that in the sham operation group was rapidly restored in a short period of time. There was a rapid increase in walking distance, however, in the operation group the increase was slower and maintained a low distance. A decrease in walking distance was observed in the operation group following surgery compared with that in the sham operation group ( $\mathrm{P}<0.05)$. BDNF, brain-derived neurotrophic factor; MOD, mean optical density.

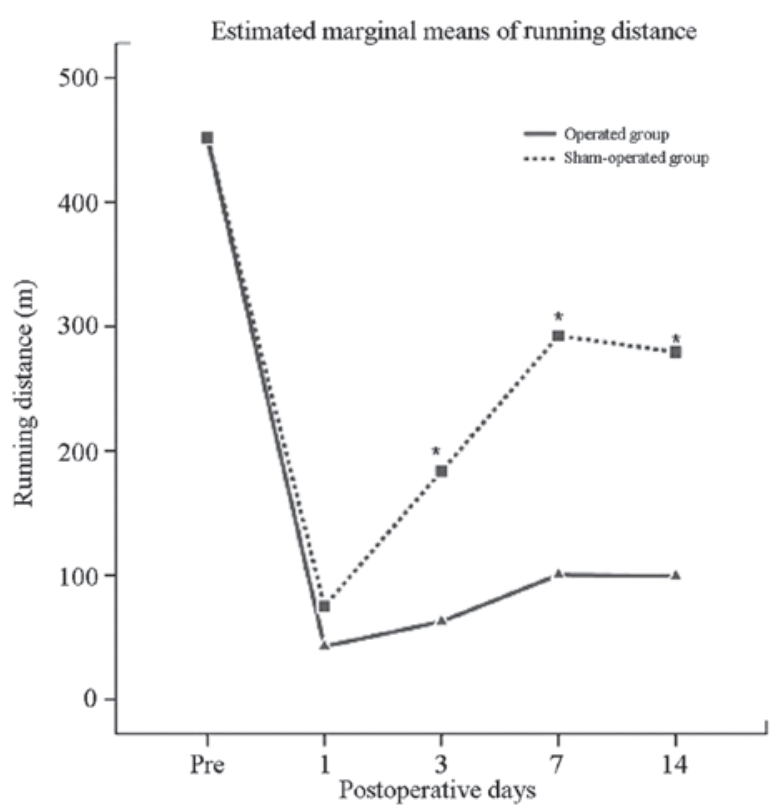

Figure 6. Correlation between BDNF expression levels and walking distance. BDNF expression levels, MOD values and walking distance in the operation group showed a significant negative correlation $(\mathrm{P}<0.05)$, but the correlativity was not close (correlation coefficient; $r=-0.55$ and $r=-0.65$ ). $\mathrm{BDNF}$, brain-derived neurotrophic factor; MOD, mean optical density.

of human body degeneration. The pressure-causing agents that compress the coccygeal nerve result in symptoms, such as intermittent claudication. Liu et al (32) effectively applied silica gel embedding to establish a rat model of LSS. We found a valid stenosis where the spinal canal was compressed by $50-70 \%$ postoperatively. This result is inconsistent with previous results used this specification (33). The reason for this difference may be that spinal canal internal diameter was not calculated effectively and directly in that study. In the present study, when measuring the walking distance of rats we identified that neurogenic intermittent claudication appeared in continuous walking; however, it showed no obvious effects on the distance measured with regard to limb sports. Yamaguchi et al applied silica gel embedding in rats and observed flat plate sports from 10 to 24 weeks post-operatively. The results of that study showed that walking distance continuously decreased as claudication increased. The pathological sections indicated epidural edema, fibrosis and axon degeneration and regeneration throughout the experiment (34).

The present study used silica gel to compress the dural sac and the walking distance in rats following surgery was observed. Finite element method with 3D software was used to measure the internal diameter of the spinal canal and showed the established LSS model in rats. This method is simple, feasible, may be manipulated and repeated, and has a high achievement ratio.

DRG contain neurons, Schwann cells, fibroblasts and satellite cells. Nerve fibers pass between all types of neurons and are divided into large, middle and small cells according to neuron diameter. DRG secretes and transports numerous types of neurotransmitters and modulators, such as BDNF, GDNF and neural cell adhesion molecule (NCAM). There are also certain receptors that have an adjusting action, such as $\gamma$-aminobutyric acid, opium, purine and a number of ion channels (35). Due to its unique action in signal transduction, DRG may be a target of therapeutic medication to decrease the generation of pain.

Stimulated DRG led to NP, which was induced by numerous different pain factors, such as opening of BDNF channel and TRPV channel, GDNF, NCAM, methionince encephalin (M-ENK), P substance, COX2, PGE2 and IL-6 (36,37). The opening of ion channels initiated a change of cell action potential, thus algesia was generated and transmitted, and its overexcitation resulted in a decreased pain threshold and hyperpathia (38).

BDNF is a member of the neurotrophin family and is important for neuronal survival and plasticity in the CNS. BDNF was first detected and purified from a pig brain in 1982 (39). BDNF has been previously demonstrated to promote numerous types of neuron growth differentiation and regeneration. It adjusts neuron apoptosis, promotes the restoration of the myelin sheath of nerve fibers, and is important in the process of synaptic maturation and plasticity. Furthermore, BDNF effects in injury, inflammatory pain and NP as pain-causing factors in Cornu dorsal medullae spinalis have been confirmed generally (40-42).

BDNF expression in different nerve cells is diverse, which may be relevant to injury time. At an acute stress state of nerve injury, BDNF is mainly synthesized and released from the Schwann cell of neurons in the middle of DRG that have a large diameter. Injury caused by chronic compression resulted in BDNF expression in neurons from the middle of DRG with a small diameter (43-45). In the present study, results of the immunohistochemistry indicated that a positive reaction occurred mainly in intracytoplasmic neurons in the middle of DRG with a small diameter. Nerve fibers also had an obvious distribution of BDNF, with only marginal expression in the 
nucleus, which is in accordance with a previous study on nerve chronic compression $(44,46)$.

The present study demonstrated that flat plate sports promoted the expression of BDNF. In injury with early stage, the upregulation expression is more obvious, and may be the result of the presence of various cell types, including big diameter cell, middle diameter cell and Schwann cell. BDNF expression levels gradually decreased with time extension, but remained higher than that of the sham operation group. This indicated that BDNF is at a state of high expression if compression is present. However, the statistical results showed that BDNF expression levels after sports were higher than those before sports. A detailed comparison at each time point between before and after sports demonstrated that the MOD value on day 3 following surgery was significantly different. The reason may be that the sample quantity is less at each time point before and after sports. Moreover, BDNF expression levels were negatively correlated with walking distance, but this correlation was not strong.

Inhibition of BDNF expression may effectively reduce pain providing a novel research direction for treating intermittent claudication clinically caused by NP. This result was confirmed in a number of animal experiments $(47,48)$. When BDNF antibody, microglia inhibitor minocycline, TrkB antagonist or p38 MAPK inhibitor were injected into the epithelium of guard or nerve ganglion, NP was effectively relieved and blocking DRG decreased symptoms, such as pain (46-50). However, BDNF not only results in pain, but is also important for the restoration and self-protection of nerves; therefore, inhibiting BDNF expression inevitably decreased nerve protection and restoration. Additional studies should be conducted to determine this role of BDNF. The present study demonstrated that BDNF expression in the LSS model was significantly upregulated following sports. Walking distance was negatively correlated with BDNF expression (walking distance decreased as BDNF expression levels increased). NP development is caused by factors, such as BDNF, as a self protection mechanism and subsequent pain restricted walking; therefore, BDNF may heighten intermittent claudication as a result of LSS.

BDNF expression increased, not only in the acute stage of injury, but also in the chronic continuous compression stage, suggesting the involvement of LSS chronic degradation process. Silica gel slices were embedded under the L5 lamina of vertebra resulting in immediate spinal stenosis, which is not in accordance with LSS chronic degradation changes. BDNF changes in the first 3 days only indicated protein expression in the acute state, but after 3 days, BDNF expression remained at a state higher than that before surgery in the sham operation group. BDNF may have continued to decrease with a longer restoration time, but results of the present have shown BDNF was at a higher state 28 days following surgery to 2 months, demonstrating that chronic continuous compression may cause a high expression of BDNF to a certain extent. The change of BDNF expression before and after sports demonstrated LSS upregulated BDNF expression during pain attack. A shorter observation time was observed with regard to flat plate sport ability, however, exercise tolerance for a longer period of time following surgery remains to be clarified. The volume of blood flow to Cauda equina may decrease during sports inducing ischemia and anoxia of the nerve root and generating inflam- mation, thereby causing pain, and the effect of the change of blood flow was not investigated in this study. In addition, this study did not show the sport ability change of rats after BDNF antibody was administrated to inhibit BDNF.

In conclusion, in the present study, silica gel was used to compress the dural sac and the sports function in rats following surgery was observed. Flat plate sports distance in the operation group was obviously less than that prior to surgery and in the sham operation group. We performed CT scanning of rats, used mimics software to set up finite element model and observed spinal canal compression at $50-70 \%$, which showed the model had been successfully established. BDNF expression levels increased in DRG in rat models of LSS and further increased following sports. The increased BDNF levels were associated with a shortened walking distance. We suggest that BDNF is important in NP generation and the transmission process in rat models of LSS. The increased BDNF expression levels indicated that BDNF may cause the pain factor of intermittent claudication, which is caused by LSS; however, simultaneouly BDNF may protect and repair nerve injury.

\section{Acknowledgements}

This study was supported by the Social Development Project of Lianyungang City (Jiangsu, China) (grant no. SH1002). We would like to thank the staff of the Experimental Animal Center, Xuzhou Medical College (Xuzhou, China).

\section{References}

1. Rainville J, Childs LA, Peña EB, et al: Quantification of walking ability in subjects with neurogenic claudication from lumbar spinal stenosis - a comparative study. Spine J 12: 101-109, 2012.

2. Okuda T, Baba I, Fujimoto Y, et al: The pathology of ligamentum flavum in degenerative lumbar disease. Spine (Phila Pa 1976) 29: 1689-1697, 2004

3. Kalichman L, Cole R, Kim DH, et al: Spinal stenosis prevalence and association with symptoms: the Framingham Study. Spine J 9: 545-550, 2009.

4. Winter CC, Brandes M, Müller C, et al: Walking ability during daily life in patients with osteoarthritis of the knee or the hip and lumbar spinal stenosis: a cross sectional study. BMC Musculoskelet Disord 11: 233, 2010.

5. Blau JN and Logue V: Intermittent claudication of the cauda equina. Lancet 1: 1081-1086, 1961.

6. Molina M, Wagner P and Campos M: Spinal lumbar stenosis: an update. Rev Med Chil 139: 1488-1495, 2011 (In Spanish).

7. Lohmander LS, Gerhardsson de Verdier M, Rollof J, Nilsson PM and Engström G: Incidence of severe knee and hip osteoarthritis in relation to different measures of body mass: a population-based prospective cohort study. Ann Rheum Dis 68: 490-496, 2009.

8. Marcol W, Kotulska K, Larysz-Brysz M and Kowalik JL: BDNF contributes to animal model neuropathic pain after peripheral nerve transection. Neurosurg Rev 30: 235-243, 2007.

9. Abbas J, Hamoud K, May H, et al: Degenerative lumbar spinal stenosis and lumbar spine configuration. Eur Spine J 19: 1865-1873, 2010.

10. Zhang H, Mei X, Zhang P, et al: Altered functional properties of satellite glial cells in compressed spinal ganglia. Glia 57: 1588-1599, 2009.

11. Wang C, Ning LP, Wang YH, et al: Nuclear factor-kappa B mediates TRPV4-NO pathway involved in thermal hyperalgesia following chronic compression of the dorsal root ganglion in rats. Behav Brain Res 221: 19-24, 2011.

12. Patte-Mensah C, Meyer L, Schaeffer V and Mensah-Nyagan AG: Selective regulation of 3 alpha-hydroxysteroid oxido-reductase expression in dorsal root ganglion neurons: a possible mechanism to cope with peripheral nerve injury-induced chronic pain. Pain 150: 522-534, 2010. 
13. Van Steenwinckel J, Noghero A, Thibault K, Brisorgueil MJ, Fischer J and Conrath M: The 5-HT2A receptor is mainly expressed in nociceptive sensory neurons in rat lumbar dorsal root ganglia. Neuroscience 161: 838-846, 2009.

14. Vranken JH: Mechanisms and treatment of neuropathic pain. Cent Nerv Syst Agents Med Chem 9: 71-78, 2009.

15. Simopoulos TT, Kraemer J, Nagda JV, Aner M and Bajwa ZH: Response to pulsed and continuous radiofrequency lesioning of the dorsal root ganglion and segmental nerves in patients with chronic lumbar radicular pain. Pain Physician 11: 137-144, 2008.

16. Boucher TJ, Okuse K, Bennett DL, Munson JB, Wood JN and McMahon SB: Potent analgesic effects of GDNF in neuropathic pain states. Science 290: 124-127, 2000.

17. Liu Y, Obata K, Yamanaka H, et al: Activation of extracellular signal-regulated protein kinase in dorsal horn neurons in the rat neuropathic intermittent claudication model. Pain 109: 64-72, 2004.

18. Ochs G, Penn RD, York M, et al: A phase I/II trial of recombinant methionyl human brain derived neurotrophic factor administered by intrathecal infusion to patients with amyotrophic lateral sclerosis. Amyotroph Lateral Scler Other Motor Neuron Disord 1: 201-206, 2000.

19. Dey ND, Bombard MC, Roland BP, et al: Genetically engineered mesenchymal stem cells reduce behavioral deficits in the YAC 128 mouse model of Huntington's disease. Behav Brain Res 214: 193-200, 2010.

20. Martin JL, Brown AL and Balkowiec A: Glia determine the course of brain-derived neurotrophic factor-mediated dendritogenesis and provide a soluble inhibitory cue to dendritic growth in the brainstem. Neuroscience 207: 333-346, 2012.

21. Cohen-Cory S, Kidane AH, Shirkey NJ and Marshak S: Brain-derived neurotrophic factor and the development of structural neuronal connectivity. Dev Neurobiol 70: 271-288, 2010.

22. Yoshii A and Constantine-Paton M: Postsynaptic BDNF-TrkB signaling in synapse maturation, plasticity, and disease. Dey Neurobiol 70: 304-322, 2010

23. Tardito D, Perez J, Tiraboschi E, Musazzi L, Racagni G and Popoli M: Signaling pathways regulating gene expression, neuroplasticity, and neurotrophic mechanisms in the action of antidepressants: a critical overview. Pharmacol Rev 58: 115-134, 2006.

24. Chan JR, Jolicoeur C, Yamauchi J, et al: The polarity protein Par-3 directly interacts with p75NTR to regulate myelination. Science 314: 832-836, 2006.

25. Ng BK, Chen L, Mandemakers W, Cosgaya JM and Chan JR: Anterograde transport and secretion of brain-derived neurotrophic factor along sensory axons promote Schwann cell myelination. J Neurosci 27: 7597-7603, 2007.

26. Kubota M, Kobayashi S, Nonoyama T, et al: Development of a chronic cervical cord compression model in rat: changes in the neurological behaviors and radiological and pathological findings. J Neurotrauma 28: 459-467, 2011.

27. Sekiguchi M, Aoki Y, Konno S and Kikuchi S: The effects of cilostazol on nerve conduction velocity and blood flow: acute and chronic cauda equina compression in a canine model. Spine (Phila Pa 1976) 33: 2605-2611, 2008.

28. Sekiguchi M, Kikuchi S and Myers RR: Experimental spinal stenosis: relationship between degree of cauda equina compression, neuropathology, and pain. Spine (Phila Pa 1976) 29 $1105-1111,2004$.

29. Watanabe K, Konno S, Sekiguchi M and Kikuchi S: Spinal stenosis: assessment of motor function, VEGF expression and angiogenesis in an experimental model in the rat. Eur Spine J 16: 1913-1918, 2007

30. Pinazo Serón MJ, Benet i Català A, Ferrer i Santaularia J, Clotas i Sancho L, Gens i Barbera M and Cartanyà i Benet A: Spinal cord compression caused by metastasis of soft tissue hepatocarcinoma. An Med Interna 16: 587-589, 1999 (In Spanish).
31. Takahashi N, Yabuki S, Aoki Y and Kikuchi S: Pathomechanisms of nerve root injury caused by disc herniation: an experimental study of mechanical compression and chemical irritation. Spine (Phila Pa 1976) 28: 435-441, 2003.

32. Liu Y, Obata K, Yamanaka H, et al: Activation of extracellular signal-regulated protein kinase in dorsal horn neurons in the rat neuropathic intermittent claudication model. Pain 109: 64-72, 2004.

33. Takenobu Y, Katsube N, Marsala M and Kondo K: Model of neuropathic intermittent claudication in the rat: methodology and application. J Neurosci Methods 104: 191-198, 2001.

34. Yamaguchi K, Murakami M, Takahashi K, Moriya $H$, Tatsuoka $\mathrm{H}$ and Chiba T: Behavioral and morphologic studies of the chronically compressed cauda equina. Experimental model of lumbar spinal stenosis in the rat. Spine (Phila Pa 1976) 24: $845-851,1999$.

35. Scholz J and Woolf CJ: The neuropathic pain triad: neurons, immune cells and glia. Nat Neurosci 10: 1361-1368, 2007.

36. St-Jacques B and Ma W: Role of prostaglandin E2 in the synthesis of the pro-inflammatory cytokine interleukin- 6 in primary sensory neurons: an in vivo and in vitro study. J Neurochem 118: 841-854, 2011.

37. Ma W: Chronic prostaglandin E2 treatment induces the synthesis of the pain-related peptide substance $\mathrm{P}$ and calcitonin gene-related peptide in cultured sensory ganglion explants. J Neurochem 115: 363-372, 2010.

38. Levin JD and Alessandri-Haber N: TRP channels: targets for the relief of pain. Biochim Biophys Acta 1772: 989-1003, 2007.

39. Lindsay RM, Barde YA, Davies AM and Rohrer H: Differences and similarities in the neurotrophic growth factor requirements of sensory neurons derived from neural crest and neural placode. J Cell Sci Suppl 3: 115-129, 1985.

40. Pezet S, Marchand F, D'Mello R, et al: Phosphatidylinositol 3-kinase is a key mediator of central sensitization in painful inflammatory conditions. J Neurosci 28: 4261-4270, 2008.

41. Merighi A, Salio C, Ghirri A, et al: BDNF as a pain modulator. Prog Neurobiol 85: 297-317, 2008.

42. Pezet S, Cunningham J, Patel J, et al: BDNF modulates sensory neuron synaptic activity by a facilitation of GABA transmission in the dorsal horn. Mol Cell Neurosci 21: 51-62, 2002.

43. Rage F, Silhol M and Tapia-Arancibia L: IL-1beta regulation of BDNF expression in rat cultured hypothalamic neurons depends on the presence of glial cells. Neurochem Int 49: 433-441, 2006.

44. Ha SO, Kim JK, Hong HS, Kim DS and Cho HJ: Expression of brain-derived neurotrophic factor in rat dorsal root ganglia, spinal cord and gracile nuclei in experimental models of neuropathic pain. Neuroscience 107: 301-309, 2001.

45. Fukuoka T, Kondo E, Dai Y, Hashimoto N and Noguchi K: Brain-derived neurotrophic factor increases in the uninjured dorsal root ganglion neurons in selective spinal nerve ligation model. J Neurosci 21: 4891-4900, 2001.

46. Li CQ, Xu JM, Liu D, Zhang JY and Dai RP: Brain derived neurotrophic factor (BDNF) contributes to the pain hypersensitivity following surgical incision in the rats. Mol Pain 4: 27, 2008.

47. Yajima Y, Narita M, Usui A, et al: Direct evidence for the involvement of brain-derived neurotrophic factor in the development of a neuropathic pain-like state in mice. J Neurochem 93: 584-594, 2005.

48. Sapunar D, Kostic S, Banozic A and Puljak L: Dorsal root ganglion-a potential new therapeutic target for neuropathic pain. J Pain Res 5: 31-38, 2012.

49. Quintão NL, Santos AR, Campos MM and Calixto JB: The role of neurotrophic factors in genesis and maintenance of mechanical hypernociception after brachial plexus avulsion in mice. Pain 136: 125-133, 2008.

50. Wang X, Ratnam J, Zou B, England PM and Basbaum AI: TrkB signaling is required for both the induction and maintenance of tissue and nerve injury-induced persistent pain. J Neurosci 29: 5508-5515, 2009. 